\title{
Differentiation of Two Types of Tryptophan Residues Related to Enzymatic Activity in a Glucoamylase from a Rhizopus sp. by $N$-Bromosuccinimide Oxidation in the Presence of Tris(hydroxymethyl)aminomethane and Maltitol
}

\author{
Masanori Iwama, Tomoko Takahashi, and Masachika IriE* \\ Department of Microbiology, Hoshi College of Pharmacy, \\ Ebara, Shinagawa-ku, Tokyo 142
}

(Received February 19, 1986)

\begin{abstract}
In order to differentiate tryptophan residues in the active site of a major glucoamylase from a

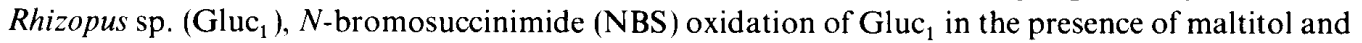
tris(hydroxymethyl)aminomethane (Tris) was studied.

1. When Gluc was $_{1}$ widized in the presence of maltitol, the hydrolytic activities of Gluc towards both soluble starch and $p$-nitrophenyl $\alpha$-D-glucopyranoside (PNPG) were scarcely protected from NBS oxidation. On the other hand, when Gluc $c_{1}$ was oxidized in the presence of Tris, the activity towards PNPG increased due to NBS oxidation of about 2 mol of tryptophan residues, whereas the activity towards maltose or soluble starch decreased.

2. The fluorescence quenching and the ultraviolet (UV) difference spectrum of Gluc induced $_{1}$ by the addition of maltitol decreased with NBS oxidation. The decreases were small in the case of Gluc $_{1}$ oxidized in the presence of maltitol, but were large and nearly parallel with the decrease in the activity towards maltose or soluble starch when Gluc $_{1}$ was oxidized in the presence of Tris.

3. It seems that at least two tryptophan residues exist in the catalytic locus of the enzyme active site, and one of them is related to the binding with a glucose moiety of substrates such as maltose but is scarcely related to the binding with the p-nitrophenyl moiety of PNPG. Another tryptophan residue seemed to be involved mainly in the catalytic action and to contribute little to maltose binding.
\end{abstract}

Keywords_-glucoamylase; Rhizopus; $N$-bromosuccinimide; tryptophan; active site

Three forms of glucoamylase [EC 3.2.1.3] have been purified from a Rhizopus species by Takahashi et al. ${ }^{1 \prime}$ As regards the active site of the major glucoamylase, Gluc ${ }_{1}$, the participation of carboxyl group(s) and tryptophan residue(s) was proved by chemical modification with 1cyclohexyl-3-(2-morpholinyl-(4)-ethyl)carbodiimide ${ }^{2)}$ and $N$-bromosuccinimide (NBS), ${ }^{3)}$ respectively. The inactivation of Gluc $_{1}$ proceeded with the oxidation of about five tryptophan residues. However, even when the enzymatic activity of Gluc $_{1}$ was destroyed by NBS oxidation, the binding ability of Gluc 1 with maltitol was fairly well retained, because fluorescence quenching of tryptophan residues induced by the addition of maltitol was quite well retained. ${ }^{3)}$ Therefore, we expected that Gluc ${ }_{1}$ has at least two tryptophan residues in or near the enzyme active site. On the other hand, we found that aminoalcohol derivatives inhibited glucoamylases, and the mode of inhibition by aminoalcohols was different from that by substrate analogues such as maltitol. ${ }^{4)}$

In this work, in order to differentiate two types of tryptophan residues in the active site of Gluc $_{1}$, NBS oxidation of Gluc $_{1}$ in the presence of two inhibitors, maltitol and tris(hydroxymethyl)aminomethane (Tris), was studied.

\section{Materials and Methods}

Chemicals_-Soluble starch was purchased from Wako Pure Chemicals (Osaka) and used as a substrate after 
exhaustive dialysis against distilled water. $p$-Nitrophenyl $\alpha$-D-glucopyranoside (PNPG) was obtained from KochLight Lab. Ltd. (Colnbrook, Bucks, England). Maltose was the product of Wako Pure Chemicals. Maltitol and Tris were purchased from Tokyo Kasei Kogyo (Tokyo) and Sigma (St. Louis, Mo., U.S.A), respectively. NBS was obtained from Nakarai Chemicals (Kyoto) and recrystallized from hot water. All other chemicals were of reagent grade.

Enzyme - - Gluc ${ }_{1}$ was purified from the commercial digestive, "Gluczyme" (from Rhizopus sp., Amano Pharm. Co., Ltd.) according to the method reported previously. ${ }^{\prime \prime}$

Determination of Glucoamylase Activity-_-(a) The enzymatic activity with soluble starch as a substrate was measured at $\mathrm{pH} 5.0$ and $37^{\circ} \mathrm{C}$ as described previously. ${ }^{1)}$ The glucose released was measured by using the "Glucose-CTest" (Wako Pure Chem.). (b) The enzymatic activity with PNPG as a substrate was measured as follows. The substrate $(10 \mathrm{~mm})$ in $1 \mathrm{ml}$ of acetate buffer $(0.1 \mathrm{M}, \mathrm{pH} 5.0)$ was hydrolyzed with $20-50 \mu$ l of enzyme solution at $37^{\circ} \mathrm{C}$. The reaction was terminated by heating the mixture at $100^{\circ} \mathrm{C}$ for $1 \mathrm{~min}$. $p$-Nitrophenol released was measured in terms of the increase in absorbancy at $400 \mathrm{~nm}$ after addition of $0.5 \mathrm{ml}$ of $0.2 \mathrm{~N} \mathrm{NaOH}$ to the reaction mixture.

NBS Oxidation of Gluc - Gluc $_{1}$ was oxidized at $25^{\circ} \mathrm{C}$ and $\mathrm{pH} 5.0$ according to the method reported previously. ${ }^{3)}$ In the case of NBS oxidation in the presence of inhibitors, specified concentrations of inhibitors were

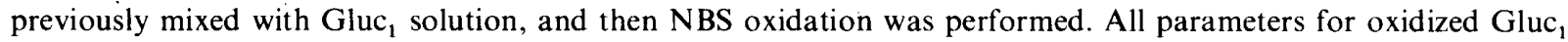
were measured after dialysis of the reaction mixture. The number of tryptophan residues oxidized was estimated from the fluorescence intensity of NBS-oxidized Gluc ${ }_{1}$ in $6 \mathrm{M}$ guanidine hydrochloride, according to the method of Pajot. ${ }^{5 \text { ) }}$

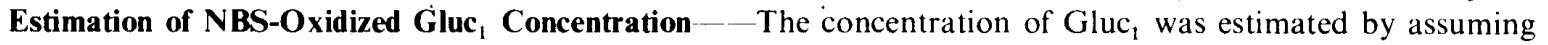

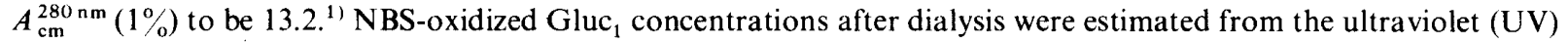
absorption at $280 \mathrm{~nm}$, which was corrected for the decrease in the UV absorbancy due to NBS oxidation before dialysis.

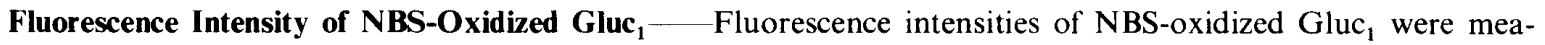
sured at $\mathrm{pH} 5.0$ (0.1 M acetate buffer) with a Shimadzu RF-502 spectrofluorometer. The enzyme fluorescence was measured at $345 \mathrm{~nm}$ with excitation at $295 \mathrm{~nm}$. The enzyme concentration used was $0.6-1.2 \mu \mathrm{M}$. The fluorescence

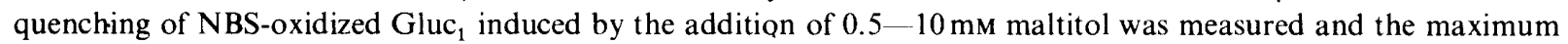
quenching value, $\Delta F_{\max }$, was calculated as the value obtained when Gluc ${ }_{1}$ was saturated with maltitol.

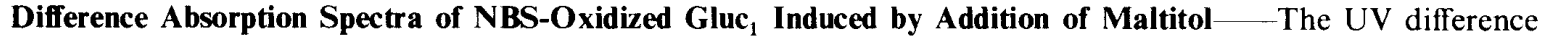

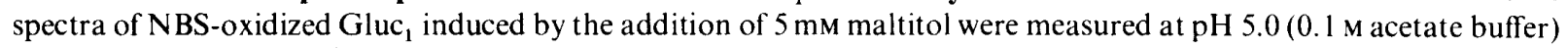
and $25^{\circ} \mathrm{C}$ with a Shimadzu UV-240 spectrophotometer using tandem cells.

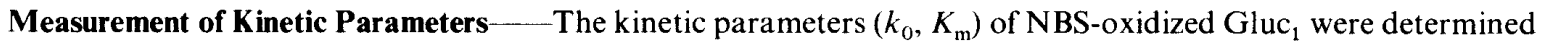
with PNPG as a substrate at pH 5.0 and 37 C. PNPG concentrations were $1.5-10 \mathrm{~mm}$. Aliquots of $50 \mu \mathrm{l}$ of $4 \quad 9 \mu \mathrm{M}$ enzyme were used for assays. Other conditions were the same as those described in Determination of Glucoamylase Activity.

\section{Results}

\section{NBS Oxidation of Gluc in $_{1}$ the Presence of Inhibitors}

Gluc ${ }_{1}$ was oxidized with various amounts of NBS in the presence and absence of maltitol $(0.6 \mathrm{M})$ or Tris $(10 \mathrm{~mm})$ at $\mathrm{pH} 5.0$ and $25 \mathrm{C}$. As shown in Fig. 1, in which the remaining activity was measured with soluble startch, maltitol slightly prevented Gluc c $_{1}$ inactivation by NBS oxidation. On the other hand, in the presence of Tris, Gluc $_{1}$ was inactivated with oxidation of

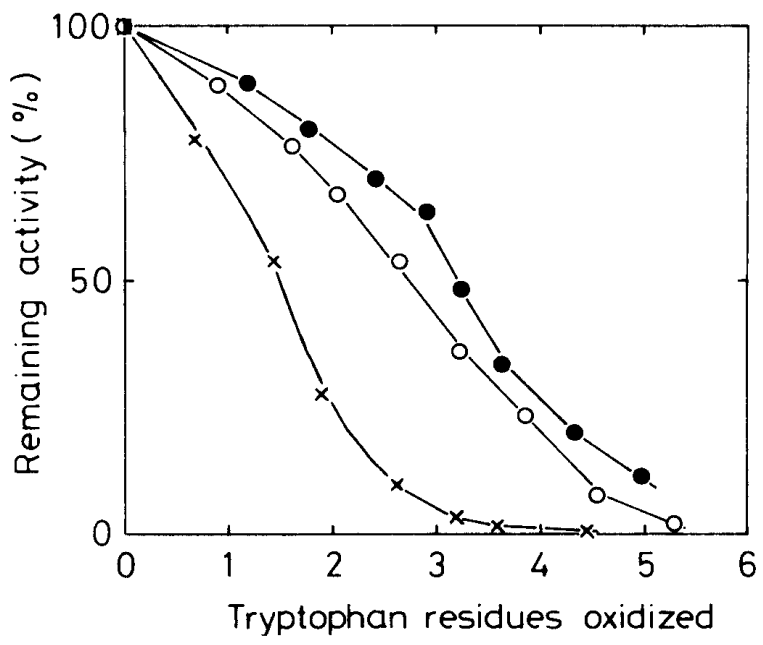

Fig. 1. NBS Oxidation of Gluc ${ }_{1}$ in the Presence of $0.6 \mathrm{~m}$ Maltitol (O), $10 \mathrm{~mm}$ Tris $(x)$, and No Inhibitor $(\mathrm{O})$

The enzyme activity was measured with soluble starch, as described in Materials and Methods. 


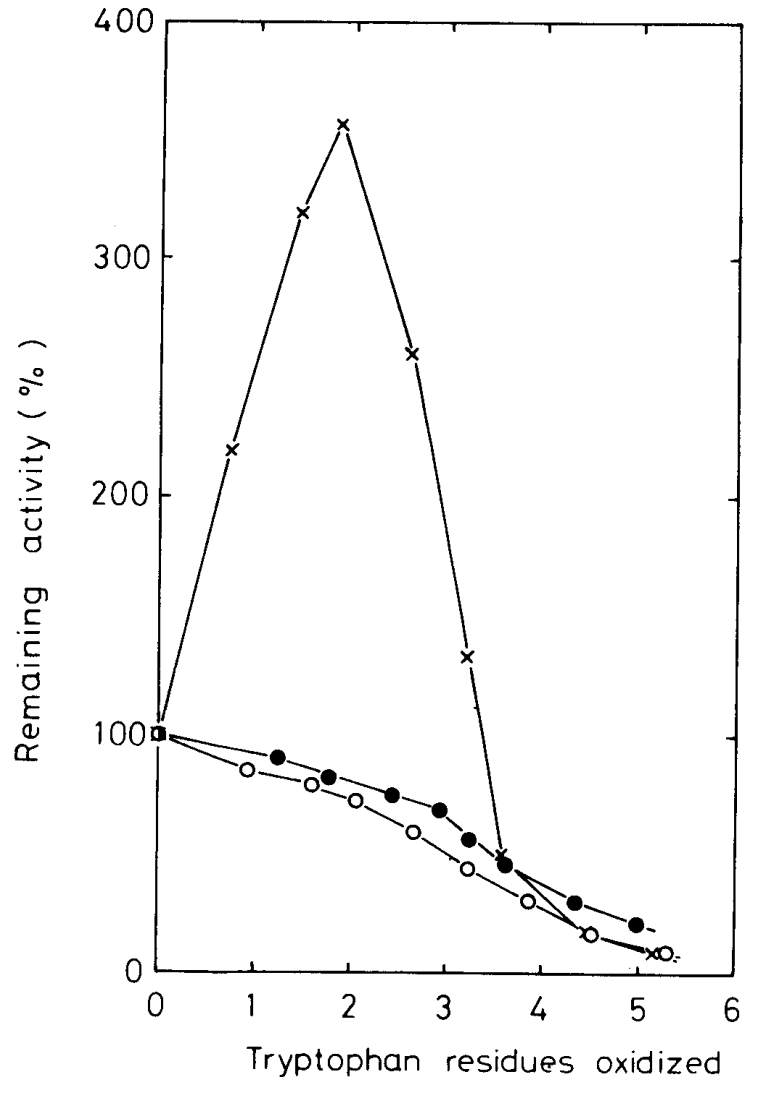

Fig. 2. The Activity of NBS-Oxidized Gluc Was Measured with $10 \mathrm{~mm}$ PNPG

The enzymatic activity was measured with PNPG, as described in Materials and Methods. The symbols are the same as Fig. 1.

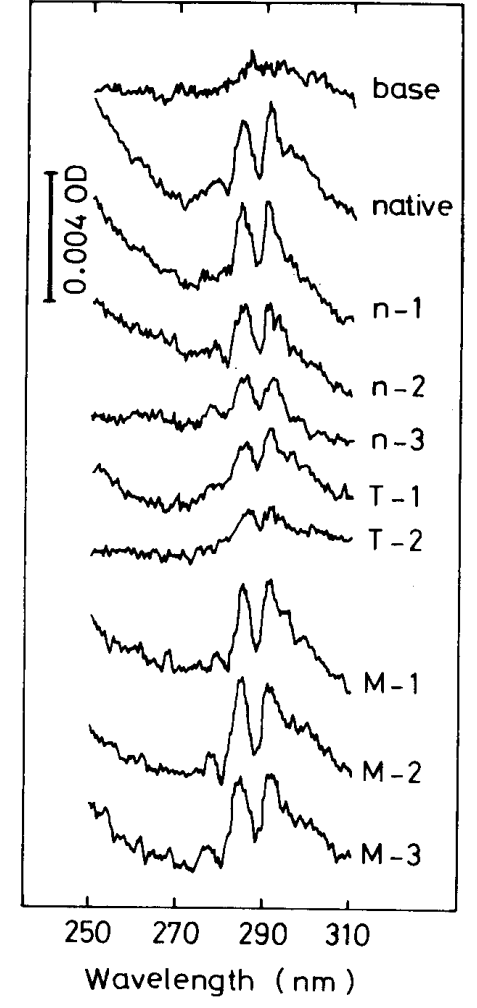

Fig. 3. The Difference UV Spectra of NBSOxidized Gluc $_{1}$ Induced by the Addition of $5 \mathrm{~mm}$ Maltitol

The enzyme concentration was $8.7 \mu \mathrm{M}$. The samples were the same as for Table I.

a smaller amount of tryptophan residues; the oxidation of about 3 tryptophan residues decreased the enzymatic activity to almost zero. These results indicated that the reactivities of tryptophan residues which were related to the glucoamylase activity seemed to be enhanced by Tris.

\section{Enzymatic Activity of NBS-Oxidized Gluc c $_{1}$ Measured with PNPG as a Substrate}

The activity of NBS-oxidized Gluc 1 was measured with $10 \mathrm{~mm}$ PNPG (Fig. 2). The samples were the same as those used for Fig. 1. The results of NBS oxidation of Gluc $c_{1}$ with and without maltitol were nearly the same as in Fig. 1, but the remaining activity of Gluc oxidized in the presence of Tris was very different from that measured with soluble starch. PNPGhydrolyzing activity was remarkably increased by oxidation of tryptophan residues up to about two, and decreased subsequently.

\section{Fluorescence Quenching of NBS-Oxidized Gluc ${ }_{1}$ on Binding of Maltitol}

The fluorescence spectra of Gluc ${ }_{1}$ oxidized in the presence and absence of maltitol or Tris were measured at $\mathrm{pH}$ 5.0. On addition of maltitol, the fluorescence intensity of oxidized Gluc ${ }_{1}$

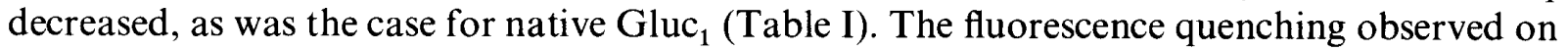
addition of maltitol to the Gluc c $_{1}$ oxidized in the presence of maltitol, and having $10 \%$ residual activity, is about $70 \%$ of $\Delta F_{\max }$ (fluorescence quenching) obtained with the native enzyme, whereas Gluc ${ }_{1}$ oxidized in the absence of maltitol, showed about $50 \%$ of $\Delta F_{\text {max. }}$. In the case of Gluc $_{1}$ oxidized in the presence of Tris, the $\Delta F_{\max }$ value decreased nearly in parallel with the remaining activity measured with soluble starch. Therefore, it could be concluded that in the 
TABLE 1. Fluorescence Intensity of NBS-Oxidized Gluc

\begin{tabular}{ccccc}
\hline \hline Sample $^{a)}$ & $\begin{array}{c}\text { Tryptophan } \\
\text { residues oxidized }\end{array}$ & $\begin{array}{c}\text { Remaining } \\
\text { activity }^{b)}\end{array}$ & $\begin{array}{c}F \\
\text { (arbitrary unit) }\end{array}$ & $\begin{array}{c}\left.\Delta F_{\max } / F_{0}{ }^{c}\right) \\
(\%)\end{array}$ \\
\hline Native & 0 & 100 & 100 & 31.7 \\
n-1 & 0.76 & 83.9 & 92.7 & 30.6 \\
n-2 & 3.58 & 31.9 & 74.3 & 20.6 \\
n-3 & 4.10 & 9.0 & 66.7 & 16.7 \\
M-1 & 1.74 & 71.7 & 93.0 & 29.5 \\
M-2 & 3.76 & 36.5 & 77.4 & 25.5 \\
M-3 & 4.28 & 10.5 & 61.4 & 22.7 \\
T-1 & 1.45 & 47.6 & 83.7 & 18.1 \\
T-2 & 4.07 & 5.5 & 63.5 & 3.4 \\
T-3 & 4.41 & 0.6 & 50.0 & - \\
\hline
\end{tabular}

a) Gluc was oxidized in the absence of inhibitor (n-1, n-2, and n-3) and in the presence of $0.6 \mathrm{M}$ maltitol $(\mathrm{M}-1, \mathrm{M}-2$, and $\mathrm{M}-3$ ) and $10 \mathrm{mM}$ Tris (T-1, T-2, and T-3). b) Remaining activity of NBS-oxidized Gluc was measured with soluble starch. The activity of the native enzyme was taken as $100 . c)$ The fluorescence quenching induced by the addition of maltitol. $\Delta F_{\max } / F_{0}$ is the ratio of $\Delta F_{\max }$ of each sample to the fluorescence intensity of the native enzyme, $F_{0}$.

TABLE II. Kinetic Parameters of NBS-Oxidized Gluc f $_{1}$ with PNPG as a Substrate

\begin{tabular}{cccc}
\hline \hline Sample & $\begin{array}{c}K_{\mathrm{m}} \times 10^{3} \\
(\mathrm{M})\end{array}$ & $\begin{array}{c}k_{0} \times 10^{2} \\
\left(\mathrm{~s}^{-1}\right)\end{array}$ & $\begin{array}{c}k_{\mathrm{o}} / K_{\mathrm{m}} \\
\left(\mathrm{s}^{-1} \cdot \mathrm{M}^{-1}\right)\end{array}$ \\
\hline Native & 2.50 & 10.4 & 41.4 \\
$\mathrm{n}-1$ & 2.59 & 8.74 & 33.8 \\
$\mathrm{n}-2$ & 2.86 & 4.57 & 16.0 \\
$\mathrm{n}-3$ & 4.00 & 2.16 & 5.4 \\
$\mathrm{M}-1$ & 2.45 & 7.92 & 32.4 \\
M-2 & 2.75 & 4.85 & 17.7 \\
M-3 & 3.00 & 2.46 & 8.2 \\
$\mathrm{~T}-1$ & 15.2 & 74.3 & 48.9 \\
$\mathrm{~T}-2$ & 23.3 & 38.3 & 16.5 \\
$\mathrm{~T}-3$ & 20.4 & 10.7 & 5.2 \\
\hline
\end{tabular}

a) The abbreviations of the enzyme samples are the same as for Table 1 .

presence of Tris, the rate of oxidation of tryptophan residue(s) which interacts with maltitol is markedly enhanced.

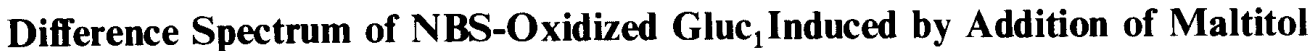

Gluc $_{1}$ produces a typical difference spectrum upon binding with substrates or analogues. ${ }^{2)}$ The difference UV spectra of NBS-oxidized Gluc ${ }_{1}$ induced by the addition of maltitol $(5 \mathrm{~mm})$ are shown in Fig. 3. The magnitude of the difference spectra decreased with NBS oxidation. In particular, the extent of the decrease was remarkable in the case of Gluc 1 oxidized in the presence of Tris. On the other hand, the magnitude of the difference spectra of Gluc $_{1}$ oxidized in the presence of maltitol was little altered. These results agreed well with those on the decrease in fluorescence quenching, as shown in Table I.

\section{Kinetic Parameters of NBS-Oxidized Gluc ${ }_{1}$}

The kinetic parameters of NBS-oxidized Gluc ${ }_{1}$ were measured with PNPG at pH 5.0 and

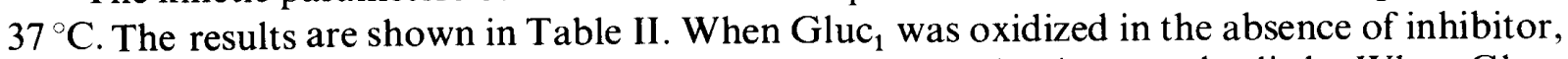
the $k_{0}$ value decreased with NBS oxidation and the $K_{\mathrm{m}}$ value increased a little. When Gluc ${ }_{1}$ 
was oxidized in the presence of maltitol, the changes in the $k_{0}$ and $K_{\mathrm{m}}$ values were very similar

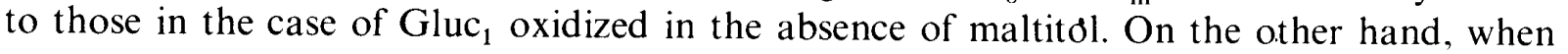
Gluc $_{1}$ was oxidized in the presence of Tris, the $K_{\mathrm{m}}$ value increased greatly, and the $k_{0}$ value also increased. As compared with the results shown in Fig. 2, the $k_{0}$ value may increase with the oxidation of up to about two tryptophan residues. When the kinetic parameters of NBSoxidized Gluc ${ }_{1}$ were measured with maltose, the $K_{\mathrm{m}}$ values were nearly constant among all the samples tested and it seemed that the decreasing activity with NBS oxidation was owing to the decreasing $k_{0}$ values (data not shown). These results indicated that the PNPG-hydrolyzing activity of Gluc $_{1}$ was protected from NBS oxidation by Tris but not by maltitol.

\section{Discussion}

In this work, we oxidized Gluc 1 with NBS in the presence of two inhibitors, maltitol and Tris, whose mechanisms of inhibition are different from each other, ${ }^{41}$ and it became clear that there were two types of tryptophan residues in $\mathrm{Gluc}_{1}$ related to the glucoamylase activity.

When Gluc 1 was oxidized with NBS in the presence of maltitol, maltitol showed a little protective effect against the inactivation of $\mathrm{Gluc}_{1}$. The fluorescence quenching and the difference UV spectrum induced by binding with maltitol indicated that the maltitol-binding ability of Gluc ${ }_{1}$ oxidized in the presence of maltitol, and having about $10 \%$ activity, was fairly well retained. Because maltitol mostly binds to subsites 2 and 3 of glucoamylases, ${ }^{6,7)}$ the tryptophan residue which can be protected by maltitol from NBS oxidation probably exists in subsite 2 or 3 , and may contribute to the binding of substrates such as maltose. It seems that the fluorescence quenching and the difference UV absorption induced by binding of substrates or analogues are mostly caused by this tryptophan residue. In this case, therefore, the decrease in enzymatic activity at the early stage of oxidation is due to the catalytic (=PNPGhydrolyzing activity) site tryptophan.

On the other hand, when Gluc 1 was oxidized with NBS in the presence of Tris, which

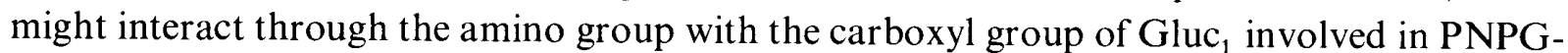
hydrolyzing activity, the catalytic site tryptophan residue was protected from oxidation. Therefore, a tryptophan residue(s) in the maltitol binding site (subsite 2 and subsite 3 ) which contributes to the enzymatic activity of Gluc $_{1}$ towards soluble starch was destroyed more markedly than in the absence of Tris. This tryptophan residue contributes to the binding with a glucose moiety, but the binding affinity of the subsite with the $p$-nitrophenyl group might be unchanged by oxidation of this tryptophan residue. Therefore, the productive binding mode of PNPG in subsites 1 and 2 might be increased relatively by NBS oxidation, and thus the PNPG-hydrolyzing activity is increased. The two tryptophan residues oxidized first in the absence of inhibitor seemed not to be related to the enzymatic activity. However, in the presence of Tris (Fig. 1), an almost linear decrease in enzymatic activity with the progress of tryptophan oxidation was observed. The results may indicate a decrease in the oxidation rates of tryptophan residues which are not related to enzymatic activity, probably induced by the interaction of Tris with subsite 1. Hiromi et al. reported that a tryptophan residue which induced the fluoresence quenching upon binding of substrates might exist in subsite 1 of the glucoamylase from Rhizopus niveus, because gluconolactone was a mixed-type inhibitor of the glucoamylase and bound with subsite 1, causing a fluorescence quenching. ${ }^{89}$ ) We suggested that this tryptophan residue may exist in subsite 2 . The discrepancy could be explained by assuming that the tryptophan residue is located in subsite 2 , though very close to subsite 1 , and is consequently affected by gluconolactone, which binds with subsite 1 .

Acknowledgement The authors wish to thank Amano Pharmaceutical Co., Ltd. for the kind gift of the enzyme source "Gluczyme". 


\section{References}

1) T. Takahashi, Y. Tsuchida, and M. Irie, J. Biochem. (Tokyo), 84, 1183 (1978).

2) M. Iwama, R. Ohtsuki, T. Takahashi, and M. Irie, J. Biochem. (Tokyo), 96, 329 (1984).

3) M. Iwama, N. Inokuchi, Y. Okazaki, T. Takahashi, A. Yoshimoto, and M. Irie, Chem. Pharm. Bull., 34, 1355 (1986).

4) M. Iwama, T. Takahashi, N. Inokuchi, T. Koyama, and M. Irie, J. Biochem. (Tokyo), 98, 341 (1985).

5) P. Pajot, Eur. J. Biochem., 63, 263 (1976).

6) T. Koyama, N. Inokuchi, Y. Kikuchi, H. Shimada, M. Iwama, T. Takahashi, and M. Irie, Chem. Pharm. Bull., 32, 757 (1984).

7) N. Inokuchi, T. Takahashi, A. Yoshimoto, and M. Irie, J. Biochem. (Tokyo), 91, 1661 (1982).

8) K. Hiromi, A. Tanaka, and M. Ohnishi, Biochemistry, 21, 102 (1982).

9) M. Ohnishi, M. Taniguchi, and K. Hiromi, Biochim. Biophys. Acta, 744, 64 (1983). 